Cite this: Nanoscale, 2013, 5, 4355

\title{
Growth and galvanic replacement of silver nanocubes in organic mediat
}

\author{
Lakshminarayana Polavarapu*a and Luis M. Liz-Marzán*abc
}

\begin{abstract}
Although metal nanoparticles with various shapes can be prepared in polar organic solvents, little has been advanced toward the shape-controlled synthesis in non-polar solvents. We report a simple method for the synthesis of nearly monodisperse single crystalline silver nanocubes in a non-polar solvent (1,2dichlorobenzene) by using oleylamine as both a reducing and capping agent. Mechanistic studies based on the time evolution of $\mathrm{Ag}$ nanoparticles revealed that multiply twinned nanocrystals form at the beginning of the reaction, which are gradually transformed into single crystalline Ag nanocubes by oxidative etching. Control experiments showed that the solvent plays an important role in the formation of such single crystalline Ag nanocubes. The effects of reaction temperature, oleylamine concentration, solvent, and the nature of the silver ion precursor on the morphology and monodispersity of the nanoparticles were systematically investigated. Additionally, the galvanic replacement reaction with $\mathrm{HAuCl}_{4}$ in an organic medium was implemented to prepare hydrophobic hollow Au-Ag nanocages with tunable localized surface plasmon resonances.
\end{abstract}

Received 11th March 2013 Accepted 17th March 2013

DOI: $10.1039 / c 3 n r 01244 a$

www.rsc.org/nanoscale solution. ${ }^{2,4,14}$ However, the size and shape controlled synthesis of $\mathrm{Au}$ and $\mathrm{Ag}$ nanoparticles with tunable optical properties in organic media is still challenging because the reduction of metal ions in organic solvents typically yields multiply twinned spherical nanoparticles due to fast homogeneous nucleation. ${ }^{15-19}$ In addition, most of the conventional precursors used for nanoparticle synthesis are inorganic salts, which are insoluble in organic solvents. ${ }^{16}$ Surfactants (containing amines or thiols) are thus required to pull such ions into organic solution by forming organometallic complexes. However, the preparation of metal nanoparticles in organic media usually has several advantages that include narrow size distribution, ${ }^{15,18}$ large scale production, ${ }^{\mathbf{1 6}}$ surface functionalization with a variety of organic capping agents, ${ }^{18}$ catalysis for organic reactions, ${ }^{20,21}$ self assembly at the interface ${ }^{3}$ and potential for the fabrication of flexible plasmonic substrates. ${ }^{22}$

In spite of its difficulty, some examples exist on the synthesis of single-crystalline ultrathin $\mathrm{Au}$ nanowires, ${ }^{\text {,23-26 }} \mathrm{Au}-\mathrm{Ag}$ bimetallic nanowires, ${ }^{27} \mathrm{Au}$ nanorod $\mathrm{s}^{28}$ and various shapes of $\mathrm{Pd}$ nanoparticle ${ }^{29}$ in organic solution. In the particular case of $\mathrm{Ag}$, there is a strong tendency to form twinned seed particles during the early stages of the reaction, both in hydrophilic and in hydrophobic solvents. ${ }^{5,19}$ It is thus difficult to prepare single crystalline $\mathrm{Ag}$ nanostructures with well defined-shapes. To overcome this problem, Xia et al. reported the polyol reduction method for the formation of single crystalline Ag nanocubes by oxidative etching of twin defects in a hydrophilic solvent (the polyol) using chloride containing salts such as $\mathrm{HCl}$ or $\mathrm{NaCl}{ }^{5}$ However, such oxidative etchants are immiscible with hydrophobic solvents, thereby limiting the synthesis of Ag nanocubes 
in non-polar organic media. To the best of our knowledge, there are only two reports in the literature for the synthesis of $\mathrm{Ag}$ nanocubes in organic media. ${ }^{\mathbf{3 0 , 3 1}}$ However, these methods are rather sensitive to the concentration of oxidative etchants and often result in a mixture of spherical and cubic Ag NPs. Peng and $\mathrm{Sun}^{31}$ reported the synthesis of $\mathrm{Ag}$ nanocubes in a binary hydrophobic solvent by using an amphiphilic oxidative etching agent, dimethyldistearylammonium chloride (DDAC), in the reaction medium. Metal salts such as $\mathrm{FeCl}_{3}$ and $\mathrm{Fe}(\mathrm{acac})_{3}$ have also been used as oxidative etching agents for the synthesis of $13 \mathrm{~nm}$ single crystalline $\mathrm{Ag}$ nanocubes in isoamyl ether, as reported by Xia et $a .^{30}$ In the above two methods, ether was chosen as the solvent to dissolve the inorganic salts as they can interact with the oxygen atom in ether.

In this work, we demonstrate a simple and optimised method for the synthesis of single crystalline Ag nanocubes in an organic solvent (1,2-dichlorobenzene (DCB)) by using oleylamine as both a reducing and capping agent, with no need for additional oxidative etching inorganic salts. We found that the compositions of the solvent and $\mathrm{Ag}$ precursor, as well as temperature, play important roles on the final morphology of the obtained $\mathrm{Ag}$ nanoparticles. Additionally, we demonstrate that $\mathrm{Ag}$ nanocubes can be transformed into $\mathrm{Au}-\mathrm{Ag}$ nanocages via a galvanic replacement reaction with $\mathrm{HAuCl}_{4}$, even in organic solvents, leading to accurate control of the LSPR position.

\section{Experimental section}

\section{Chemicals}

Silver nitrate $\left(\mathrm{AgNO}_{3}\right)$, silver acetate $\left(\mathrm{CH}_{3} \mathrm{COOAg}\right)$, silver trifluoro acetate $\left(\mathrm{CF}_{3} \mathrm{COOAg}\right), \mathrm{HAuCl}_{4} \cdot 3 \mathrm{H}_{2} \mathrm{O}$, oleylamine (OA) (technical grade, 70\%), chlorobenzene, 1-octadecene and toluene were purchased from Sigma-Aldrich. 1,2-Dichloro benzene (DCB; 99\% extra pure) was purchased from Acros Organics. All chemicals were used as received. Caution: DCB is highly toxic and should be handled with care in an appropriate fume hood.

\section{Synthesis of silver nanocubes}

$0.6 \mathrm{mmol}$ of $\mathrm{AgNO}_{3}$ and $6 \mathrm{mmol}$ of oleylamine were dissolved in $50 \mathrm{~mL}(0.44 \mathrm{~mol})$ of DCB and the reaction mixture was sonicated until $\mathrm{AgNO}_{3}$ was completely dissolved. The solution colour changed from colourless to pale yellow upon sonication. The resulting solution was transferred into an oil bath and the bath temperature was slowly raised up to $165{ }^{\circ} \mathrm{C}$ at a rate of $5{ }^{\circ} \mathrm{C}$ $\min ^{-1}$ and then the reaction solution gradually acquired a deep yellow colour. The reaction mixture was kept for 48 hours at $165{ }^{\circ} \mathrm{C}$ with continuous stirring under atmospheric conditions and then slowly cooled down to room temperature. The resulting solution contained nearly monodisperse single crystalline silver nanocubes. To study the time evolution of $\mathrm{Ag}$ nanoparticles during the reaction, small aliquots were taken from the reaction mixture at different reaction times by using a Pasteur pipette and analysed by UV-visible spectroscopy and transmission electron microscopy (TEM). Control experiments were carried out using the same procedure to investigate the effect of temperature $\left(120,140,165\right.$ and $185{ }^{\circ} \mathrm{C}$ ), solvent (toluene, chlorobenzene, and octadecene), and amount of surfactant ( $3 \mathrm{mmol}, 12 \mathrm{mmol}$ ) on the morphology of the obtained Ag nanoparticles. The effect of precursor on the morphology of the obtained Ag NPs was tested by carrying out the reaction $\left(0.88 \mathrm{~mol}, \mathrm{DCB}, 0.6 \mathrm{mmol}\right.$ of $\mathrm{AgNO}_{3}$ and $6 \mathrm{mmol}$ of oleylamine) using $\mathrm{CH}_{3} \mathrm{COOAg}$ and $\mathrm{CF}_{3} \mathrm{COOAg}$ as precursors.

It was found that the reaction time and yield of $\mathrm{Ag}$ nanocubes strongly depends on the reaction volume. When the reaction was carried out in $100 \mathrm{~mL}(0.88 \mathrm{~mol})$ of DCB, while keeping the amount of oleylamine and $\mathrm{AgNO}_{3}$ constant, the reaction was completed in $12 \mathrm{~h}$ and the monodispersity and yield of $\mathrm{Ag}$ nanocubes were increased. However, when the reaction was carried out in $25 \mathrm{~mL}(0.22 \mathrm{~mol})$ of DCB, the product mainly contained twinned $\mathrm{Ag}$ nanoparticles with a small percentage of $\mathrm{Ag}$ nanocubes, even after $48 \mathrm{~h}$ of reaction time.

\section{Galvanic replacement reaction}

In a typical synthesis, $1 \mathrm{~mL}$ of the as prepared (using $0.6 \mathrm{mmol}$ $\mathrm{AgNO}_{3}, 6 \mathrm{mmol}$ oleylamine and $0.88 \mathrm{~mol}$ DCB) silver nanocube solution was injected into a round-bottom flask containing $19 \mathrm{~mL}$ of toluene under magnetic stirring and the system was heated to $100{ }^{\circ} \mathrm{C}$ in an oil bath. A fresh $\mathrm{HAuCl}_{4}$ stock solution was prepared by dissolving $9 \mathrm{mg}$ of $\mathrm{HAuCl}_{4} \cdot 3 \mathrm{H}_{2} \mathrm{O}$ in $6 \mathrm{~mL}$ of toluene and $0.4 \mathrm{~mL}$ of oleylamine and stored in the dark before use. Different volumes $(0.1,0.15,0.2,0.25,0.3,0.35,0.4$ and $0.8 \mathrm{~mL}$ ) of the $\mathrm{HAuCl}_{4}$ stock solution were added to the boiling reaction mixture under magnetic stirring, so that the final $\mathrm{Au}$ : Ag molar ratios were 6\%, 9\%, 12\%, 15\%, 18\%, 21\%, 24\% and $50 \%$, respectively. After each addition of $\mathrm{HAuCl}_{4}$ the stirring was continued for another $10 \mathrm{~min}$ until the UV-Vis spectra did not change further. Galvanic replacement was also carried out at room temperature to study the effect of reaction temperature, while keeping the other parameters unchanged. The obtained particles were purified by centrifugation and redispersed in toluene for TEM characterization.

\section{Characterization}

The Ag nanoparticles were characterized by UV-visible spectroscopy (Agilent 8543, $1 \mathrm{~cm}$ path length quartz cuvettes) and TEM (using a JEOL JEM 2010F, field emission electron microscope, operating at $200 \mathrm{kV}$ ). Energy-dispersive X-ray spectroscopy (EDS) spectra were obtained by using an INCA X-Sight spectrometer (Oxford instruments), within the TEM instrument. For TEM analysis, the obtained Ag nanocubes were first purified by centrifugation and re-suspended in toluene. Then, a drop of the purified nanoparticle solution was spotted onto a carbon coated copper grid and dried in a fume hood at room temperature.

\section{Results and discussion}

\section{Growth of Ag nanocubes}

The synthesis of hydrophobic Ag nanocubes is based on the reduction of $\mathrm{AgNO}_{3}$ with oleylamine at high temperature in DCB solution (see Experimental section for details). Fig. 1e shows a 

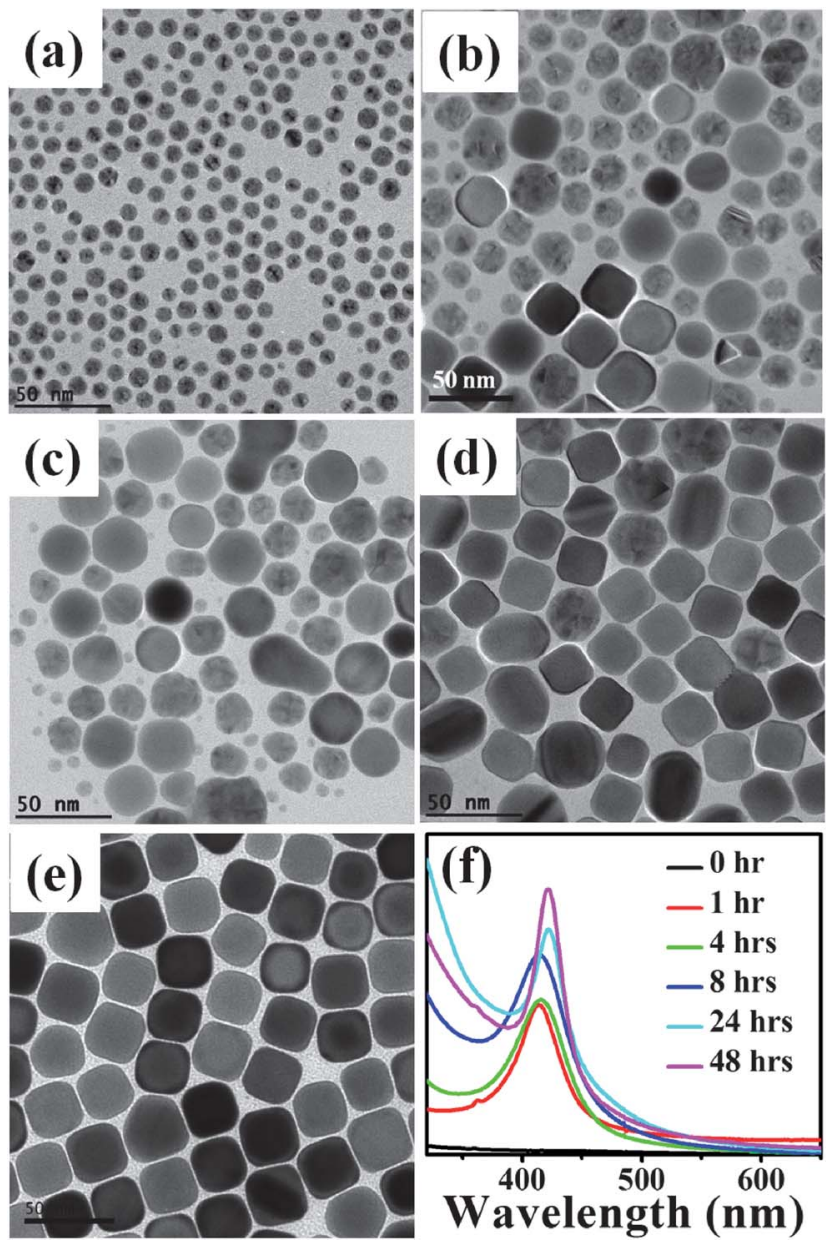

Fig. 1 TEM images of the Ag nanoparticles obtained at (a) 1, (b) 4, (c) 8, (d) 24 and (e) 48 hours of reaction time in $0.44 \mathrm{~mol}$ of DCB solution $\left(0.6 \mathrm{mmol}\right.$ of $\mathrm{AgNO}_{3}$ and $6 \mathrm{mmol}$ of oleylamine) and their corresponding extinction spectra (f). HRTEM image of the $\mathrm{Ag}$ nanoparticles formed after $1 \mathrm{~h}$ of reaction time is shown in Fig.S2.†

typical TEM image of $\mathrm{Ag}$ nanocubes obtained after $48 \mathrm{~h}$ of reaction in $0.44 \mathrm{~mol}$ of DCB solution $\left(0.6 \mathrm{mmol}\right.$ of $\mathrm{AgNO}_{3}$ and $6 \mathrm{mmol}$ of oleylamine), clearly showing nearly monodisperse $\mathrm{Ag}$ nanocubes with slight truncation and $26 \pm 2 \mathrm{~nm}$ average edge length (standard deviation 1.6\%). Additional TEM images obtained under lower magnification confirm the monodispersity, while HRTEM shows that they are single crystalline (see Fig. S1 $\dagger$ ). The slight truncation of the Ag nanocubes arises due to partial oxidation and release of $\mathrm{Ag}$ atoms at the corners during the synthesis, but the cubic shape is preserved upon storage in non-polar solvents for extended periods of time (at least 10 months). This is in contrast with the tendency of $\mathrm{Ag}$ nanocubes prepared by polyol reduction, which tend to become increasingly rounded when they are stored in water for shorter periods of time. ${ }^{32}$

To investigate the growth mechanism responsible for the formation of single crystalline $\mathrm{Ag}$ nanocubes, we monitored their growth by extracting aliquots from the reaction mixture at different reaction times and analysing them by UV-visible spectroscopy and TEM (after diluting $10 \mu \mathrm{L}$ aliquots in $2 \mathrm{~mL}$ of toluene). Fig. 1 shows representative TEM images of Ag nanoparticles obtained at different reaction times, as well as their corresponding extinction spectra. As shown in Fig. 1a, the Ag nanoparticles formed after one hour of reaction are spherical, with sizes ranging from 8 to $10 \mathrm{~nm}$ and with the corresponding extinction maximum at $414 \mathrm{~nm}$. High resolution TEM images (Fig. S2†) revealed a multiply twinned structure for these nanoparticles. As the reaction progresses to $t=4 \mathrm{~h}$, multiply twinned quasi spherical Ag nanoparticles with a wider size distribution (10-20 nm) coexist with a small population of single crystalline polyhedral Ag nanoparticles, including nanocubes, as observed in the TEM images (Fig. 1b). During this initial step, the extinction spectrum broadened due to a broader particle size distribution and the LSPR maximum redshifted to $416 \mathrm{~nm}$, which is attributed to an increase in both particle size and anisotropy. As the reaction proceeds further, the population of twinned particles decreases whereas the population of single crystalline polyhedral nanoparticles and nanocubes gradually increases, as reflected in the TEM images (Fig. 1c-d). The sample obtained after $24 \mathrm{~h}$ (Fig. 1d) essentially consists of single crystalline $\mathrm{Ag}$ nanocubes with a small percentage of multiply twinned particles and the corresponding extinction spectrum exhibits a red-shifted sharp LSPR centred at $422 \mathrm{~nm}$, corresponding to $\mathrm{Ag}$ nanocubes of $\sim 26 \mathrm{~nm}$ lateral size. These results suggest that the size of the initially obtained multiply twinned $\mathrm{Ag}$ nanoparticles increases by continuous reduction of $\mathrm{Ag}^{+}$ions by oleylamine, but apparently the growth stops after reaching a critical size and then the nanoparticles start to transform into single crystalline nanoparticles by oxidative etching of defect sites. As shown in Fig. 1d, the size of the twinned particles formed after $24 \mathrm{~h}$ is the same as that of the single crystal nanocubes. When the reaction time was further extended to $48 \mathrm{~h}$, all the twinned particles were completely converted into single crystal nanocubes (a histogram of the estimated population distribution of twinned vs. single crystalline NPs as a function of reaction time is shown in Fig. S3†) and thus the final product essentially contains truncated nanocubes of $\sim 26 \mathrm{~nm}$ average edge length, with an LSPR of $422 \mathrm{~nm}$ and a shoulder at $362 \mathrm{~nm}$ (Fig. 1e and f), which are attributed to a dipolar plasmon resonance and a quadrupolar mode, respectively. ${ }^{33-35}$ Regarding the observed crystallinity change from multiply twinned nanoparticles to single crystals, it has been previously reported for the synthesis of $\mathrm{Ag}$ nanocubes in aqueous solutions, in relation with the oxidative etching of highly reactive defect regions by etchants such as $\mathrm{HCl}$ or $\mathrm{NaCl}^{\mathbf{5 , 1 2}}$ In the present organic phase synthesis, additional oxidative etchants were not added, but $\mathrm{NO}_{3}{ }^{-}$and $\mathrm{Cl}^{-}$ions from the silver salt and the solvent (DCB), as well as dissolved oxygen, could initiate the slow oxidative etching and dissolution of defect sites at a relatively high temperature and prolonged reaction time, thereby leading to the formation of the final single crystalline Ag nanocubes. ${ }^{5}$ In previous works it has been reported that $\mathrm{NO}_{3}{ }^{-}$and $\mathrm{Cl}^{-}$ions could initiate the etching of twinned Ag NPs and their transformation into single crystalline NPs in the absence of dissolved oxygen. ${ }^{31,36}$ We tested this hypothesis by means of a control synthesis in which the reaction mixture was purged by bubbling nitrogen gas and then 
reaction was carried out under nitrogen while the other experimental parameters were the same as indicated in Fig. 1. TEM characterization (Fig. S4 $\dagger$ ) showed that most of the obtained NPs after $48 \mathrm{~h}$ of reaction are $\mathrm{Ag}$ nanocubes, with a small population of twinned NPs and nanorods. These results suggest that etching can indeed occur in the absence of oxygen, but the presence of some twinned Ag NPs indicates the reaction was slower. However, when the reaction was carried out in atmospheric conditions all the twinned Ag NPs were converted into single crystalline Ag nanocubes (Fig. S1†).

\section{Effect of reaction parameters}

The reducing ability of oleylamine and the oxidative etching capability of $\mathrm{NO}_{3}{ }^{-}$and $\mathrm{Cl}^{-}$ions usually increase with increasing the reaction temperature. ${ }^{31}$ Therefore, we studied the effect of reaction temperature on the morphology of the $\mathrm{Ag}$ nanoparticles by running the reaction at different temperatures $(120$, 140,165 and $185^{\circ} \mathrm{C}$ ) while other parameters were the same as those used for the experiment described in Fig. 1. Representative TEM images are shown in Fig. 2, from which we found that the optimum temperature for the synthesis of $\mathrm{Ag}$ nanocubes was $165{ }^{\circ} \mathrm{C}$ (Fig. 2c). When the temperature was further increased up to $185{ }^{\circ} \mathrm{C}$, the product contained a mixture of nanocubes, nanospheres and few nanorods (Fig. 2d), which might be related to more extensive etching and truncation of the nanocubes.

A similar effect was observed when the amount of oleylamine was decreased to $3 \mathrm{mmol}$ (Fig. S5a†). In this case, insufficient protection of the Ag nanocubes surface may lead to further truncation into spherical nanoparticles. However, when more oleylamine was used $(12 \mathrm{mmol})$, polydisperse MT nanoparticles were formed (Fig. S5b†), which is likely due to strong protection of the initially formed NPs, preventing the etching of defect sites.

Interestingly, the nature of the solvent was found to play a significant role on the morphology of the obtained nanoparticles. Indeed, when solvents such as toluene, chlorobenzene and octadecene were used (Fig. S6†), an increase in the
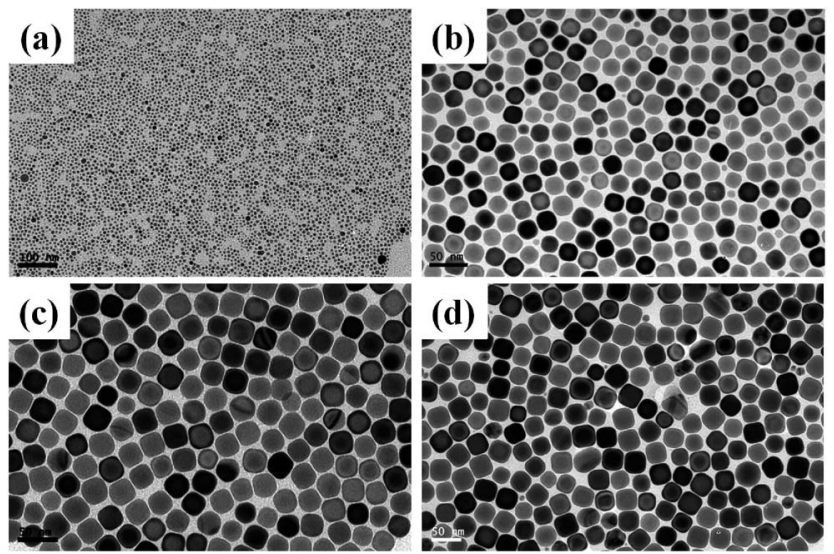

Fig. 2 TEM images of the Ag nanoparticles synthesized at different reaction temperatures: (a) 120, (b) 140, (c) 165 and (d) $185^{\circ} \mathrm{C}$, while other reaction conditions were kept the same as in Fig. $1\left(0.6 \mathrm{mmol}\right.$ of $\mathrm{AgNO}_{3}$ and $6 \mathrm{mmol}$ of oleylamine in $0.44 \mathrm{~mol}$ of DCB solution).
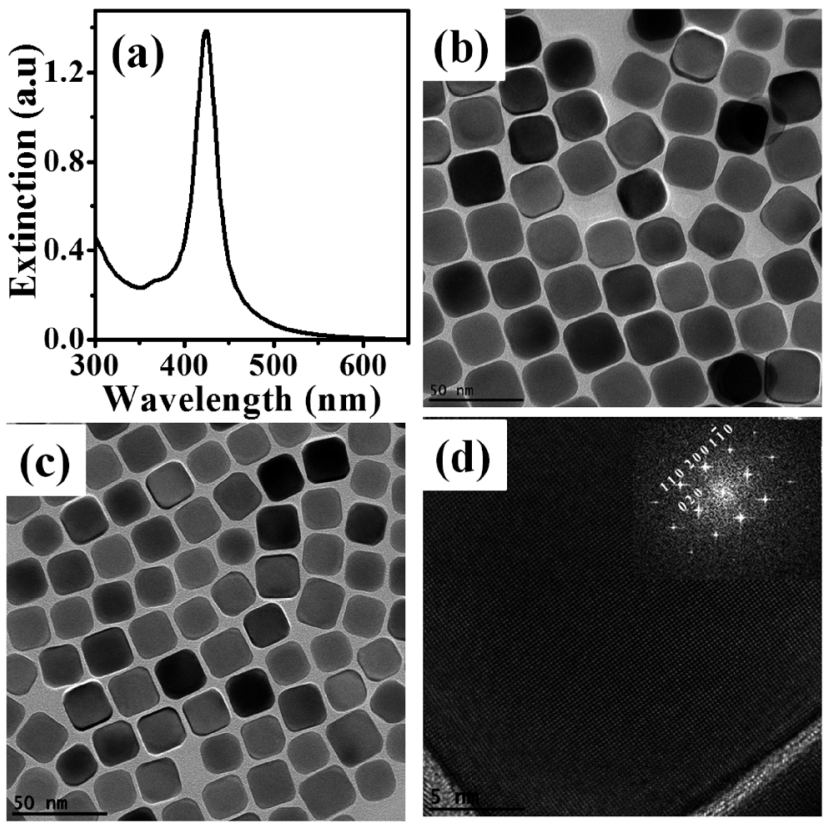

Fig. 3 ( $a$ and $b$ ) Extinction spectrum and TEM image of nanocubes prepared in 0.88 moles of DCB solution by $\mathrm{Ag} \mathrm{NO}_{3}$ reduction. (c) TEM image of nanocubes prepared in 0.88 moles of $\mathrm{DCB}$ solution using $\mathrm{CF}_{3} \mathrm{COOAg}$ as a precursor. Other reaction parameters were the same as those in Fig. $1\left(0.6 \mathrm{mmol}_{\mathrm{AgNO}_{3}}, 6 \mathrm{mmol}\right.$ oleylamine). (d) HRTEM image of a Ag nanocube. The inset is the FFT spot pattern, indicating highly symmetric spots, which can be indexed to (110), (200) and (020) reflections of face-centred cubic silver.

reaction yield was noted for high boiling point solvents (Fig. S6a†). However, the Ag nanoparticles obtained in such solvents were polycrystalline and did not display cubic morphology. The main difference between DCB and the other solvents is a higher content of chloride atoms as well as a lower $\mathrm{C}-\mathrm{Cl}$ bond dissociation energy of DCB as compared to chlorobenzene, ${ }^{37}$ resulting in a higher tendency to release chloride ions at high temperature, which can eventually etch the twinned Ag nanoparticles. ${ }^{5,12}$

In addition, the influence of reactants concentration was studied by carrying out the synthesis in different amounts ( 0.22 and 0.88 moles) of DCB, while keeping the amounts of $\mathrm{AgNO}_{3}$ and oleylamine the same as in the experiment described in Fig. 1. The extinction spectra and TEM images of the obtained $\mathrm{Ag}$ nanoparticles in different amounts of DCB are summarized in Fig. S7. $†$ The results indicate that $\mathrm{Ag}$ nanocubes with higher quality and yield can be prepared by reducing $0.6 \mathrm{mmol}$ of $\mathrm{AgNO}_{3}$ with $6 \mathrm{mmol}$ of oleylamine in 0.88 moles of DCB (Fig. 3b and S7d†). Fig. 3a shows the extinction spectra of the Ag nanocubes obtained in 0.88 moles of $\mathrm{DCB}$, with a dipolar LSPR at $424 \mathrm{~nm}$ and a quadrupolar band at $365 \mathrm{~nm} \cdot{ }^{34}$ The TEM image (Fig. 3b) clearly shows uniform contrast over each nanocube, suggesting that they are monocrystalline, which was confirmed by HRTEM on individual particles (Fig. $3 \mathrm{~d}$ and S8†). Finally, the reaction was tested with other silver ion precursors such as $\mathrm{CF}_{3} \mathrm{COOAg}$ and $\mathrm{CH}_{3} \mathrm{COOAg}$. Monodisperse $\mathrm{Ag}$ nanocubes with $23 \mathrm{~nm}$ average edge length were obtained using $\mathrm{CF}_{3} \mathrm{COOAg}$ (Fig. 3c), with high monodispersity and yield 
(Fig. S9†). In contrast, $\mathrm{CH}_{3} \mathrm{COOAg}$ produced quasi spherical $\mathrm{Ag}$ nanoparticles (Fig. S10†).

\section{Galvanic replacement and formation of Au nanocages}

Galvanic replacement reactions have been an effective strategy for the preparation of nanostructures, in which two metals are exchanged because of the difference in their electrochemical potentials, so that a hole is generated due to oxidative dissolution of metal nanocrystals, which subsequently act as templates. ${ }^{38-40}$ This strategy has been widely applied to the reaction (in polar solvents) of $\mathrm{Ag}$ nanoparticles of various shapes with $\mathrm{HAuCl}_{4}$ to synthesize hollow nanoparticles with tuneable optical properties. ${ }^{\mathbf{3 8 , 4 0 , 4 1}}$ In this reaction, Ag reacts with $\mathrm{HAuCl}_{4}$ at an atomic ratio of $3: 1$. However, application of this process in organic media has been restricted to spherical $\mathrm{Ag}$ nanoparticles, mainly due to the limitations in the synthesis of shape controlled $\mathrm{Ag}$ nanoparticles in these solvents. ${ }^{\mathbf{4 2 - 4 4}} \mathrm{We}$ evaluated the application of the galvanic replacement reaction to our Ag nanocubes in a non-polar environment, at different reaction temperatures. Shown in Fig. 4 are representative TEM images of the $\mathrm{Ag}$ nanocubes before and after titrating with different amounts of $\mathrm{HAuCl}_{4}$ solution in toluene $0.1,0.2,0.25$ and $0.35 \mathrm{~mL}$, so that the final $\mathrm{Au}: \mathrm{Ag}$ molar ratios were $6 \%$, $12 \%, 15 \%$ and $21 \%$, respectively. The reaction was carried out at $100{ }^{\circ} \mathrm{C}$. It is clear from the images that the initial single crystalline $\mathrm{Ag}$ nanocubes (edge length $\mathrm{ca} .24 \mathrm{~nm}$ ) readily display signs of galvanic replacement reaction (through a hole on one of their faces) upon addition of a small amount ( $6 \mathrm{~mol} \%)$ of $\mathrm{HAuCl}_{4}$ (Fig. 4b), resembling the usual galvanic replacement reactions in aqueous solution. ${ }^{38}$ For larger amounts of $\mathrm{HAuCl}_{4}$, the void size gradually increased, while the corners became further truncated, likely because of dealloying, until ultimately the particles were converted into cage like structures (Fig. 4d). From the TEM images, the particles appear to have different morphology, which is due to the orientation of NPs on either (100) or (111) facets, depending on their degree of corner truncation, ${ }^{45}$ and HRTEM characterization indicates that the nanocages retained the monocrystallinity of the original cubes (Fig. 4; see also Fig. S11 $\nmid$ for a larger image). The composition of the nanocages was confirmed by EDS on a single particle, to contain $\mathrm{Au}$ and $\mathrm{Ag}$, probably as an alloy (Fig. S12†). Interestingly, upon further addition of $\mathrm{HAuCl}_{4}$, well defined voids were observed at the corners, so that most of the particles appeared to display a nanoframe morphology. The average size of the nanoparticles slightly increased up to $28 \mathrm{~nm}$, indicating the deposition of gold atoms on the exterior surfaces as $\mathrm{Ag}$ was depleted from the interior, but the monodispersity was preserved (see Fig. S13 $†$ ). The stability of the obtained nanocages in nonpolar solvents, as well as their high surface-tovolume ratio are expected to render them very useful as catalysts for organic reactions. It is important to note however that excess addition of $\mathrm{HAuCl}_{4}([\mathrm{Au}]:[\mathrm{Ag}]=50 \mathrm{~mol} \%)$ leads to the fragmentation of $\mathrm{Au}$ nanostructures (Fig. S14†). We additionally found that when the galvanic replacement reaction was carried out at room temperature, rather different morphologies were obtained, where small protuberances formed from the initial stages, finally leading to irregular Au nanoframes (Fig. S15†). We speculate that the reason behind this different reshaping process is the decrease of interdiffusion rate of $\mathrm{Au}$ and $\mathrm{Ag}$ atoms at room temperature.

The morphological and composition changes involved in the galvanic replacement reaction lead to changes in the LSPR of the particles, so that the reaction could be monitored by UV-visible spectroscopy. Fig. 5 shows a photograph of the dispersions of $\mathrm{Ag}$ nanoparticles in toluene, before and after galvanic replacement
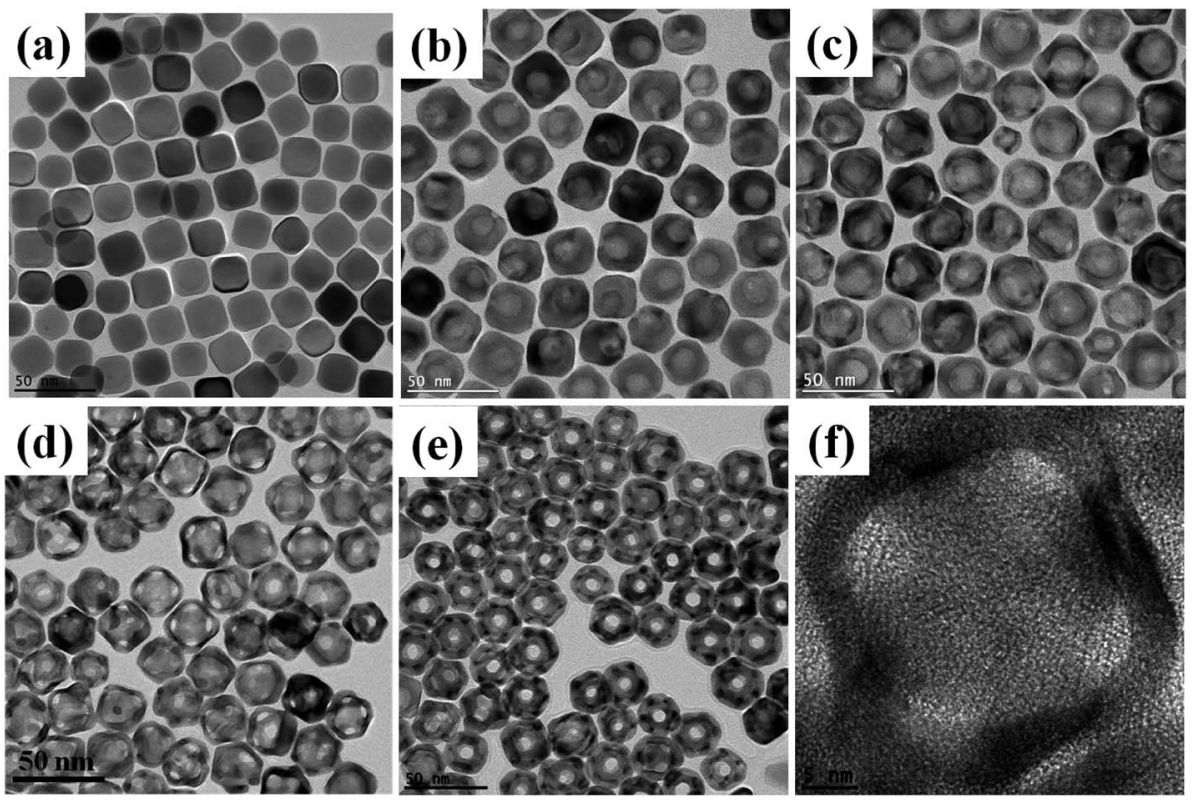

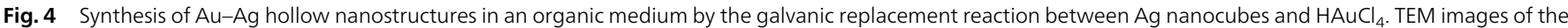

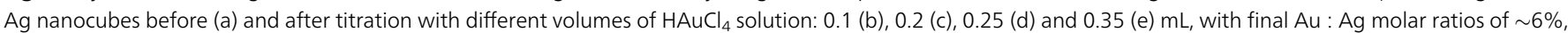
$12 \%, 15 \%$, and $21 \%$, respectively. (f) HRTEM image of one Au-Ag nanocage from (d), see Fig. S11 for a large size image. $\dagger$ 


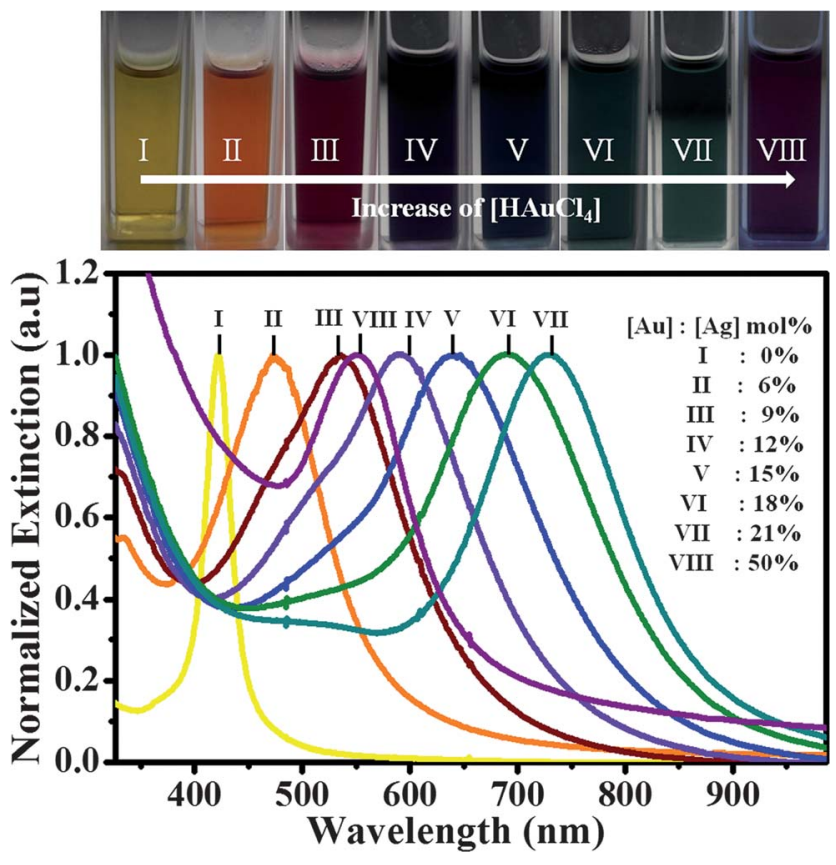

Fig. 5 Photograph and normalized extinction spectra of nanocube dispersions before (I) and after (II-VIII) galvanic replacement with different volumes of $\mathrm{HAuCl}_{4}: 0.1,0.15,0.2,0.25,0.3,0.35$ and $0.8 \mathrm{~mL}$, with final $\mathrm{Ag}$ : Au molar ratios of $\sim 6 \%, 9 \%, 12 \%, 15 \%, 18 \%, 21 \%, 50 \%$, respectively. The spectra prior to normalization are shown in Fig. S16.†

with different amounts of $\mathrm{HAuCl}_{4}$, along with their normalized extinction spectra. The colour of the dispersions changes as yellow-orange-red-violet-blue-green-pink with increasing amounts of $\mathrm{HAuCl}_{4}$ and such colour changes are reflected in the significant LSPR redshifts (Fig. 5; see also the extinction spectra prior to normalization in the ESI, Fig. S16†). However, the extinction spectra are rather narrow, indicating that the obtained particles are quasi-monodisperse, as evidenced by TEM (Fig. 4). Again, excess addition of $\mathrm{HAuCl}_{4}$ leads to a blue shift due to fragmentation of the hollow nanostructures.

\section{Conclusion}

We have developed a simplified method for the preparation of single-crystalline $\mathrm{Ag}$ nanocubes in organic solvents by using oleylamine as a reducing and capping agent. The growth mechanism was studied by means of time-dependent TEM and UVvisible spectroscopy characterization. It was found that twinned seed particles are formed at early stages, which eventually transform into single crystalline $\mathrm{Ag}$ nanocubes by preferential oxidative etching of twinned particles. Furthermore, galvanic replacement reaction between $\mathrm{Ag}$ nanocubes and $\mathrm{HAuCl}_{4}$ in an organic medium was successfully accomplished, leading to the formation of hollow alloy nanostructures (nanocages and nanoframes) with distinct LSPR peaks, in agreement with similar studies in polar solvents. We expect that the high quality of these hydrophobic Ag nanocubes and AgAu hollow nanostructures will be very useful for a number of applications such as catalysis, photonics, nonlinear optics and sensing.
This work has been funded by the European Research Council (ERC Advanced Grant \# 267867, PLASMAQUO). We thank Jorge Pérez-Juste, Isabel Pastoriza-Santos, Akshaya Kumar Samal, Ana Sánchez-Iglesias, and Stefanos Mourdikoudis for helpful discussions. Marco Möller is acknowledged for assistance with HRTEM and EDS characterization.

\section{Notes and references}

1 L. M. Liz-Marzan, Langmuir, 2006, 22, 32-41.

2 M. Grzelczak, J. Perez-Juste, P. Mulvaney and L. M. LizMarzan, Chem. Soc. Rev., 2008, 37, 1783-1791.

3 A. Sanchez-Iglesias, M. Grzelczak, J. Perez-Juste and L. M. Liz-Marzan, Angew. Chem., Int. Ed., 2010, 49, 99859989.

4 Y. G. Sun and Y. N. Xia, Science, 2002, 298, 2176-2179.

5 B. Wiley, T. Herricks, Y. G. Sun and Y. N. Xia, Nano Lett., 2004, 4, 1733-1739.

6 S. P. Zhang, K. Bao, N. J. Halas, H. X. Xu and P. Nordlander, Nano Lett., 2011, 11, 1657-1663.

7 W. H. Wang, Q. Yang, F. R. Fan, H. X. Xu and Z. L. Wang, Nano Lett., 2011, 11, 1603-1608.

8 H. J. Feng, Y. M. Yang, Y. M. You, G. P. Li, J. Guo, T. Yu, Z. X. Shen, T. Wu and B. G. Xing, Chem. Commun., 2009, 1984-1986.

9 P. Christopher, H. L. Xin and S. Linic, Nat. Chem., 2011, 3, 467-472.

10 S. De, T. M. Higgins, P. E. Lyons, E. M. Doherty, P. N. Nirmalraj, W. J. Blau, J. J. Boland and J. N. Coleman, ACS Nano, 2009, 3, 1767-1774.

11 B. Sepulveda, P. C. Angelome, L. M. Lechuga and L. M. LizMarzan, Nano Today, 2009, 4, 244-251.

12 S. H. Im, Y. T. Lee, B. Wiley and Y. N. Xia, Angew. Chem., Int. Ed., 2005, 44, 2154-2157.

13 I. Pastoriza-Santos and L. M. Liz-Marzan, Langmuir, 2002, 18, 2888-2894.

14 M. R. Langille, M. L. Personick, J. Zhang and C. A. Mirkin, J. Am. Chem. Soc., 2012, 134, 14542-14554.

15 M. Chen, Y. G. Feng, X. Wang, T. C. Li, J. Y. Zhang and D. J. Qian, Langmuir, 2007, 23, 5296-5304.

16 L. Polavarapu, K. K. Manga, K. Yu, P. K. Ang, H. D. Cao, J. Balapanuru, K. P. Loh and Q. H. Xu, Nanoscale, 2011, 3, 2268-2274.

17 L. Polavarapu and Q. H. Xu, Nanotechnology, 2009, 20, 185606-185612.

18 H. Hiramatsu and F. E. Osterloh, Chem. Mater., 2004, 16, 2509-2511.

19 Y. Sun, Chem. Soc. Rev., 2013, 42, 2497-2511.

20 H. Lee, S. E. Habas, S. Kweskin, D. Butcher, G. A. Somorjai and P. D. Yang, Angew. Chem., Int. Ed., 2006, 45, 7824-7828.

21 X. Hong, D. Wang, S. Cai, H. Rong and Y. Li, J. Am. Chem. Soc., 2012, 134, 18165-18168.

22 L. Polavarapu and L. M. Liz-Marzan, Phys. Chem. Chem. Phys., 2013, 15, 5288-5300.

23 Z. Y. Huo, C. K. Tsung, W. Y. Huang, X. F. Zhang and P. D. Yang, Nano Lett., 2008, 8, 2041-2044. 
24 X. M. Lu, M. S. Yavuz, H. Y. Tuan, B. A. Korgel and Y. N. Xia, J. Am. Chem. Soc., 2008, 130, 8900-8901.

25 N. Pazos-Perez, D. Baranov, S. Irsen, M. Hilgendorff, L. M. Liz-Marzan and M. Giersig, Langmuir, 2008, 24, 9855-9860.

26 C. Wang, Y. J. Hu, C. M. Lieber and S. H. Sun, J. Am. Chem. Soc., 2008, 130, 8902-8903.

27 X. Hong, D. S. Wang, R. Yu, H. Yan, Y. Sun, L. He, Z. Q. Niu, Q. Peng and Y. D. Li, Chem. Commun., 2011, 47, 5160-5162.

28 Z. Q. Li, J. Tao, X. M. Lu, Y. M. Zhu and Y. N. Xia, Nano Lett., 2008, 8, 3052-3055.

29 Z. Q. Niu, Q. Peng, M. Gong, H. P. Rong and Y. D. Li, Angew. Chem., Int. Ed., 2011, 50, 6315-6319.

30 Y. Y. Ma, W. Y. Li, J. Zeng, M. McKiernan, Z. X. Xie and Y. N. Xia, J. Mater. Chem., 2010, 20, 3586-3589.

31 S. Peng and Y. G. Sun, Chem. Mater., 2010, 22, 6272-6279.

32 Q. A. Zhang, W. Y. Li, L. P. Wen, J. Y. Chen and Y. N. Xia, Chem.-Eur. J., 2010, 16, 10234-10239.

33 A. Bottomley, D. Prezgot, A. Staff and A. Ianoul, Nanoscale, 2012, 4, 6374-6382.
34 M. A. Mahmoud and M. A. El-Sayed, J. Phys. Chem. C, 2008, 112, 14618-14625.

35 Y. H. Lee, H. Chen, Q.-H. Xu and J. Wang, J. Phys. Chem. C, 2011, 115, 7997-8004.

36 Y. Tang and M. Ouyang, Nat. Mater., 2007, 6, 754-759.

37 X. H. Li, Z. X. Tang and X. Z. Zhang, J. Struct. Chem., 2009, 50, 34-40.

38 Y. Sun and Y. Xia, J. Am. Chem. Soc., 2004, 126, 3892-3901.

39 Y. Sun and Y. Wang, Nano Lett., 2011, 11, 4386-4392.

40 M. R. Jones, K. D. Osberg, R. J. Macfarlane, M. R. Langille and C. A. Mirkin, Chem. Rev., 2011, 111, 3736-3827.

41 S. E. Skrabalak, J. Y. Chen, Y. G. Sun, X. M. Lu, L. Au, C. M. Cobley and Y. N. Xia, Acc. Chem. Res., 2008, 41, 1587-1595.

42 X. Lu, H.-Y. Tuan, J. Chen, Z.-Y. Li, B. A. Korgel and Y. Xia, J. Am. Chem. Soc., 2007, 129, 1733-1742.

43 Y. Yin, C. Erdonmez, S. Aloni and A. P. Alivisatos, J. Am. Chem. Soc., 2006, 128, 12671-12673.

44 Karvianto and G. M. Chow, J. Nanopart. Res., 2012, 14, 1186.

45 J. Chen, J. M. McLellan, A. Siekkinen, Y. Xiong, Z.-Y. Li and Y. Xia, J. Am. Chem. Soc., 2006, 128, 14776-14777. 